\section{Neuron type-specific miRNA represses two broadly expressed genes to modulate an avoidance behavior in C. elegans}

\author{
Tanja Drexel, Katharina Mahofsky, \\ Richard Latham, Manuel Zimmer, \\ and Luisa Cochella
}

Research Institute of Molecular Pathology (IMP), Vienna
Biocenter (VBC), 1030 Vienna, Austria

Two broad gene classes are distinguished within multicellular organisms: cell type-specific genes, which confer particular cellular properties, and ubiquitous genes that support general cellular functions. However, certain socalled ubiquitous genes show functionally relevant cell type-specific repression. How such repression is achieved is poorly understood. MicroRNAs (miRNAs) are repressors, many of which are expressed with high cell type specificity. Here we show that mir-791, expressed exclusively in the $\mathrm{CO}_{2}$-sensing neurons in Caenorhabditis elegans, represses two otherwise broadly expressed genes. This repression is necessary for normal neuronal function and behavior of the animals toward $\mathrm{CO}_{2}$. miRNA-mediated repression of broadly transcribed genes is a previously unappreciated strategy for cellular specialization.

Supplemental material is available for this article.

Received July 25, 2016; revised version accepted August 29, 2016.

The ability to regulate different sets of genes to generate and maintain distinct cell types with diverse morphologies and functions is the basis for multicellularity. Evolutionary studies suggest that this ability derived from an ancestral multifunctional eukaryotic cell expressing a relatively high number of genes. Evolution to multicellularity relied on the segregation of functions from that primordial cell into an increasingly diversified number of descendants (Arendt 2008). Functional segregation as well as acquisition of new cell-specific functions resulted in genes expressed in specific cell types, while others remained broadly or even ubiquitously expressed. The addition of repressors of gene expression must have been key during this process, restricting the execution of genetic programs to specific cell types.

It has long been recognized that even so-called ubiquitous genes are regulated in specific cells or conditions. For example, the housekeeping gene encoding the lactate/pyruvate transporter MCT1 is specifically repressed in pancreatic $\beta$ cells to achieve a correct cellular and organismal response to changes in glucose concentration (Ishihara et al. 1999). However, mechanisms for cell-spe-

[Keywords: miRNAs; cell identity; C. elegans; carbon dioxide] Corresponding author: cochella@imp.ac.at

Article published online ahead of print. Article and publication date are online at http://www.genesdev.org/cgi/doi/10.1101/gad.287904.116. Freely available online through the Genes \& Development Open Access option. cific repression of broadly expressed genes remain relatively unexplored.

MicroRNAs (miRNAs) are a broad class of post-transcriptional repressors that expanded with the onset of multicellularity (Berezikov 2011). Since miRNA evolution is more dynamic than that of protein-coding genes and since many miRNAs are expressed with high spatiotemporal specificity (e.g., see Aboobaker et al. 2005; Wienholds et al. 2005), they are proposed to contribute to the gene expression profiles of specific cell types. While several examples illustrate the roles of miRNAs in refining expression patterns of other spatiotemporally restricted genes, broadly expressed housekeeping genes tend to avoid miRNA-binding sites in their $3^{\prime}$ untranslated regions (UTRs) (Stark et al. 2005). However, it is conceivable that miRNAs present exclusively in a given cell type can repress broadly transcribed genes in those cells. Specific repression could provide unique features to specialized cells while preserving the function of such broadly transcribed targets in the rest of the organism. Moreover, post-transcriptional regulation provides an appealing solution to regulate housekeeping genes, whose transcription is constrained by the compact nature of their promoters (Zeitlinger and Stark 2010; Zabidi et al. 2015).

Supporting this hypothesis, we show that mir-791 is expressed exclusively in three pairs of sensory neurons in the nematode Caenorhabditis elegans that are essential for the avoidance response to high $\mathrm{CO}_{2}$ (Bretscher et al. 2011). We found that mir-791 represses two target genes that are transcribed in all types of somatic cells, consistent with their proposed functions in general cell biology: a PKA anchor protein (akap-1 [A kinase anchor protein 1]) and a carbonic anhydrase (cah-3). miRNA-mediated repression of these targets in the $\mathrm{CO}_{2}$-sensing neurons is necessary for normal neuronal physiology and, ultimately, the animal's response to this critical environmental cue.

\section{Results and Discussion \\ mir-791 is necessary for a normal response of C. elegans to $\mathrm{CO}_{2}$}

We conducted a screen for miRNAs present in specific C. elegans neurons using reporters with $\sim 40 \mathrm{~kb}$ of genomic sequence in fosmid vectors, which accurately recapitulate endogenous expression patterns (Tursun et al. 2009). We found mir-791 exclusively in three pairs of sensory neurons, which, based on position, morphology, and expression of known markers, were identified as the BAG, AFD, and ASE pairs of neurons (Fig. 1A; Supplemental Fig. S1A). These neurons are the main cells responsible for sensing elevated $\mathrm{CO}_{2}$ and triggering the escape response in C. elegans (Bretscher et al. 2011), as high $\mathrm{CO}_{2}$ is toxic and is proposed to signal the presence of predators (Bretscher et al. 2008; Hallem and Sternberg 2008; Sharabi et al. 2009). Expression of mir-791 begins in embryos shortly after the neurons' birth and continues throughout adulthood (Supplemental Fig. S1A; our small RNA sequencing data not shown). Given the specificity of mir-

(C) 2016 Drexel et al. This article, published in Genes \& Development, is available under a Creative Commons License (Attribution 4.0 International), as described at http://creativecommons.org/licenses/by/4.0/. 
A

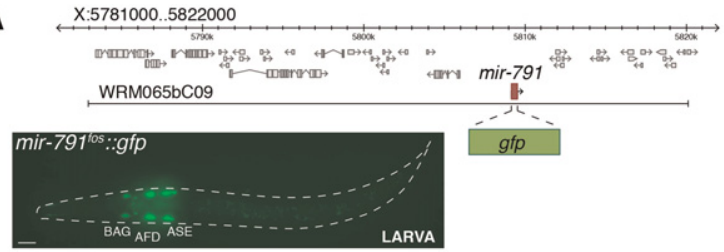

B
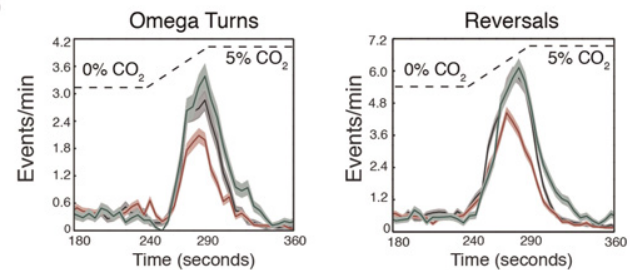

$=\mathrm{N} 2$ $=$ mir-791(0) $=\operatorname{mir}-791(0)$ Ex mir-791
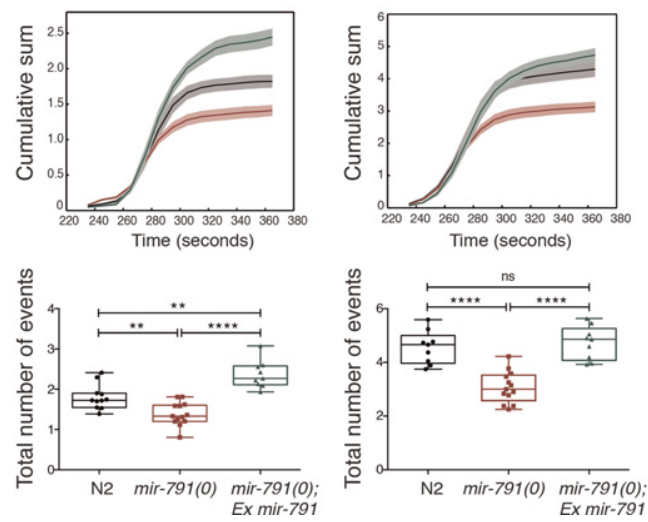

Figure 1. mir-791 is expressed in the $\mathrm{CO}_{2}$-sensing neurons of C. elegans and is required for the response to $\mathrm{CO}_{2}$. $(A)$ Schematic of the fosmid-based reporter used to monitor mir-791 expression and a representative image of a transgenic animal; the three pairs of neurons are labeled. Bar, $10 \mu \mathrm{m}$. (B, top) Population means (lines) and standard errors of the means (shading) of turning and reversal rates for wild type (N2), mir-791(0), and mir-791(0) with extrachromosomal copies of mir-791 during a gradual increase of $\mathrm{CO}_{2}$ from $0 \%$ to $5 \%$. (Middle) Cumulative sum of all of the turning or reversal events during the stimulation phase (240-360 sec). (Bottom) Box and whiskers representation of the total number of turns and reversals during the stimulation phase (each data point is an endpoint of the individual replicate used to calculate the averages shown above). $n=9 \mathrm{~N} 2 ; n=13$ mir791(0); $n=9$ mir-791(0), Ex mir-791. Each replicate is an experiment with 50-100 animals. Boxes show interquartile range, and whiskers indicate full range. $\left.\left(^{* * * *}\right) P<0.0001 ;{ }^{* * *}\right) P<0.001 ;\left(^{* *}\right) P<0.01 ;\left(^{*}\right) P$ $<0.05$; (ns) not significant, Mann-Whitney test.

791 expression, we hypothesized that it may be involved in $\mathrm{CO}_{2}$ sensing in C. elegans.

We used the escape response of C. elegans from high $\mathrm{CO}_{2}$ as a proxy for the ability of the worms to sense changes in $\mathrm{CO}_{2}$ levels (Bretscher et al. 2008; Hallem and Sternberg 2008). C. elegans responds to diverse stimuli by modulating the frequency of reorientation maneuvers (Pierce-Shimomura et al. 1999), such as switching from forward to backward-directed crawling (reversal) and performing sharp-angle turns ( $\Omega$ turn). Worms use a single reversal-turning sequence to acutely escape aversive stimuli (Chalfie et al. 1985). To test the role of mir-791 in the $\mathrm{CO}_{2}$ evoked escape behavior, we deleted the miRNA locus using CRISPR/Cas9 and compared these animals with the wild type in their response to a gradual increase of $\mathrm{CO}_{2}$ from $0 \%$ to $5 \%$. Upon this stimulus, wild-type animals robustly increase their frequency of reversals and turns. An- imals lacking mir-791 display a significant reduction in these maneuvers in response to the same stimulus (Fig. 1B), while their average speed modulation is the same as wild type (Supplemental Fig. S2). mir-791-deficient animals have a similar onset of response to $\mathrm{CO}_{2}$ but stop responding to the stimulus before their wildtype counterparts, an effect best visualized by displaying the cumulative sum of turns or reversals over time (Fig. $1 \mathrm{~B}$, middle). To confirm that this effect is due to loss of mir-791, we reintroduced mir-791 under its endogenous promoter as an extrachromosomal multicopy transgene and found that this rescued the worm's response to $\mathrm{CO}_{2}$ (Fig. 1B). In fact, it is likely that overexpression of mir791 from this transgene increased the locomotor response of these animals to levels higher than wild type.

mir-791 has been linked in a family with mir-790 due to their identical seed sequences (Supplemental Fig. S1B). In addition, mir-790 is also expressed in the $\mathrm{CO}_{2}$-sensing neurons (Supplemental Fig. S1C), suggesting that they could act redundantly. However, using a deletion allele that we generated for mir-790 by itself or in a double-mutant combination with mir-791(0), we showed that this is not the case (Supplemental Fig. S1D-F). Overall, we found that a single miRNA, mir-791, which is expressed exclusively in the $\mathrm{CO}_{2}$-sensing neurons of $C$. elegans, is required for the normal behavioral response of the animals to $\mathrm{CO}_{2}$.

mir-791 is required mainly in the BAGs and functions specifically in $\mathrm{CO}_{2}$ sensing

While mir-791 is transcribed in three pairs of $\mathrm{CO}_{2}$-sensing neurons, the BAGs have the highest contribution to the avoidance behavior (Bretscher et al. 2011). To test whether the role of mir-791 was more significant in BAG than in AFD or ASE, we expressed mir-791 under promoters specific for each of these neurons in mir-791(0) animals. Reintroduction of mir-791 in BAG alone was sufficient to recover the response to elevated $\mathrm{CO}_{2}$ to a large extent, while exclusive expression in AFD or ASE alone did not rescue the mir-791(0) defect (Supplemental Fig. S3). mir791 likely contributes to all three neuron pairs, but its role in the BAG neurons accounts for the largest fraction of the measurable phenotype. Hence, we focused on these neurons for further characterization.

To test whether mir-791 acts specifically in $\mathrm{CO}_{2}$ sensing or more generally affects BAG function, we took advantage of the fact that these neurons also sense decreases in $\mathrm{O}_{2}$ concentration (Zimmer et al. 2009). Using the same behavioral setup, we asked whether mir-791(0) animals have a defect in their response to changes in $\mathrm{O}_{2}$ while keeping $\mathrm{CO}_{2}$ at $0 \%$. In this assay, animals lacking mir-791 respond to $\mathrm{O}_{2}$ changes indistinguishably from wild type (Supplemental Fig. S4). We conclude that mir791 plays a specific role in $\mathrm{CO}_{2}$ sensing and is not generally required for other sensory functions of the BAGs. Also, as mir-791(0) animals show normal basal speed in the absence of stimuli and since normal frequency of turns and reversals upon $\mathrm{O}_{2}$ decreases, we ruled out a general locomotion defect in these animals.

mir-791 is not required for the basic cellular identity of the BAGs but is required for neuronal physiology

As mir-791 is expressed from the time the $\mathrm{CO}_{2}$ sensory neurons are born, we asked whether it plays a role in 
specifying the identity of these cells. To do so, we scored the expression of functionally relevant terminal markers of these neurons in animals with or without mir-791. The candidate $\mathrm{CO}_{2}$ sensor in the BAGs is a receptor guanylate cyclase encoded by gcy-9 (Hallem et al. 2011; Smith et al. 2013). Upon $\mathrm{CO}_{2}$ increase, GCY-9 is thought to generate cGMP, opening a cGMP-gated channel formed by the products of $\operatorname{tax}-2$ and $\operatorname{tax}-4$. We generated a fosmidbased reporter for gcy-9 and used available reporters for tax-4 and also gcy-33, a soluble guanylate cyclase involved in $\mathrm{O}_{2}$ sensing (Zimmer et al. 2009), and flp-17, a neuropeptide secreted by the BAGs (Ringstad and Horvitz 2008). All of these reporters were expressed indistinguishably in the BAG neurons in the presence or absence of mir791 (Fig. 2A). Also, the morphology and position of the neurons were unaffected. This is in line with the partial decrease in the behavioral response to $\mathrm{CO}_{2}$ in mir-791(0) animals as compared with animals in which the BAGs are ablated (Bretscher et al. 2011). Together, this supports that mir-791 is not required for specifying the basic identity of the BAGs and implies that targets of mir-791 might impact the physiology of these cells.

To monitor the physiological response of the BAGs, we measured their $\mathrm{CO}_{2}$-evoked activity using the genetically encoded fluorescent calcium indicator GCaMP6f /Chen et al. 2013). Upon the same $\mathrm{CO}_{2}$ increase as above, the BAG neurons responded reliably in wild-type animals with a biphasic profile of activity followed by a gradual decline even though $\mathrm{CO}_{2}$ was still high, suggesting that the neurons become desensitized to the stimulus (Fig.
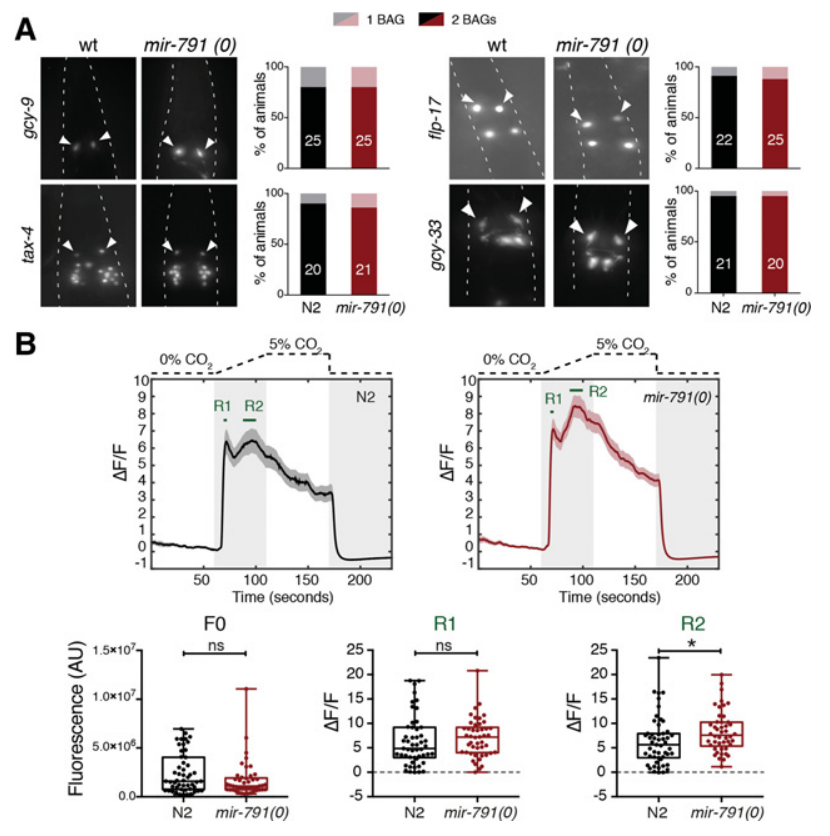

Figure 2. mir-791 is not required for expression of core BAG genes but is necessary for a wild-type response to $\mathrm{CO}_{2}$. (A) Representative fluorescence images of animals expressing reporters for functionally important genes in the BAG neurons in wild type (wt; N2) and mir791(0). For the transgenes used, see Supplemental Table S2. Twenty or more animals of each genotype were scored for the presence of each reporter (shown in the bar graphs). (B) Mean and SEM of changes in fluorescence of GCaMP6f in the BAG neurons in response to changes in $\mathrm{CO}_{2} . n=51 \mathrm{~N} 2 ; n=46$ mir-791(0). Statistics (Mann-Whitney test) on the baseline (F0), initial (R1), and sustained (R2) responses are shown.
2B). Animals lacking mir-791 had a similar initial response but a slightly higher and more sustained second phase of activity (Fig. 2B; Supplemental Fig. S5). The biphasic increase in fluorescence reflects the BAG response to $\mathrm{CO}_{2}$, as both phases are abolished in animals lacking the $\mathrm{CO}_{2}$ sensor GCY-9 (Supplemental Fig. S5C). While we cannot rule out additional effects downstream from $\mathrm{Ca}^{2+}$ influx, this suggests that mir-791 has an effect on the $\mathrm{Ca}^{2+}$ response of the BAGs to $\mathrm{CO}_{2}$. Whether this differential response explains the behavioral defect remains to be tested, but it suggests the possibility that sustained BAG activity may somehow limit the measured behavioral response.

akap-1 and cah-3 are broadly expressed but are specifically repressed by mir-791 in the BAGs

TargetScanWorm 6.2 (Jan et al. 2011) provided us with a short list of likely mir-791 targets. Among the top 10 predicted targets, there were five genes with multiple mir791-binding sites whose protein products suggested possible links to $\mathrm{CO}_{2}$ sensing or neuronal signaling (Supplemental Table S1).

The top two predicted targets were particularly interesting. AKAP-1 is a member of a protein family initially found to tether the cAMP-dependent kinase PKA to distinct cellular compartments, generating subcellular signaling domains. However, AKAPs also bind phosphatases, phosphodiesterases, and other regulators of cyclic nucleotide signaling (Langeberg and Scott 2015). We hypothesized that AKAP-1 could affect cyclic nucleotide signaling downstream from GCY-9. The second predicted target, cah-3, encodes a carbonic anhydrase (CA). These enzymes catalyze the reversible hydration of $\mathrm{CO}_{2}$ to produce $\mathrm{HCO}_{3}{ }^{-}$and $\mathrm{H}^{+}$and are essential to maintain $\mathrm{CO}_{2}$, electrolyte, and $\mathrm{pH}$ homeostasis (Supuran 2008). In addition, CAs have been implicated in $\mathrm{CO}_{2}$ sensing in multiple systems (Luo et al. 2009; Cummins et al. 2013).

To investigate the expression of these genes, we generated fosmid-based fluorescent reporters (Supplemental Fig. S6; Tursun et al. 2009). To facilitate visualization and quantification, we inserted a T2A peptide followed by GFP:H2B at the $\mathrm{C}$ terminus of each of the genes such that two independent polypeptides were made: untagged AKAP-1 or CAH-3 and nuclear GFP:H2B. Production of both proteins is under regulation by the wild-type $3^{\prime}$ UTR. Both reporters are broadly expressed in most tissues of worms. akap-1 is expressed from early embryos until adulthood and in most cells of the animal, both soma and germline (Fig. 3A; Supplemental Fig. S6A). Its homolog in Drosophila, spoonbill, is also ubiquitously expressed in flies of all stages (http://www.flybase.org; http://www. fruitfly.org). The cah-3 reporter is also broadly expressed in all major somatic cell types (Fig. 3B; Supplemental Fig. S6B). The broad expression of these two genes is in line with their proposed general cellular functions.

In the BAG neurons, however, GFP fluorescence from both reporters was barely detectable (Fig. 3). To test whether the low-level expression of cah-3 and akap-1 in the BAGs is due to mir-791, we measured GFP intensity in these cells in wild-type or mir-791(0) backgrounds. The absence of mir-791 resulted in the derepression of both akap-1 and cah-3 reporters in the BAGs to levels similar to neighboring reference cells that do not express mir791 (Fig. 4A,B). In addition, removing all seed-matching 
A
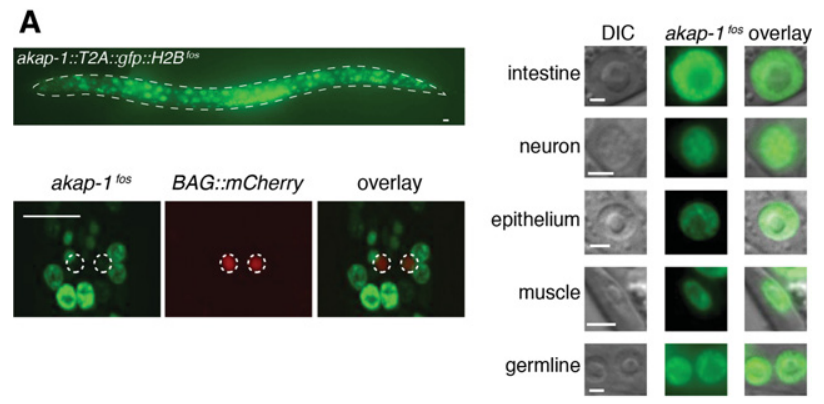

B
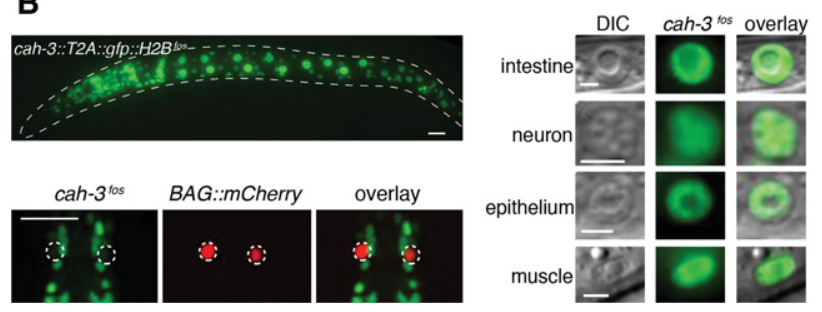

Figure 3. AKAP-1 and CAH-3 are expressed in most cell types of the animal but are low or absent in the BAG neurons. Representative fluorescence images of animals carrying akap-1 $(A)$ and cah-3 $(B)$ nuclearlocalized fosmid-based reporters (Supplemental Fig. S6). (Right) Zoom in of the different nuclear types (distinguished by size and morphology) expressing both reporters. Bars, $2 \mu \mathrm{m}$. (Bottom left) Focus on the BAG nuclei localized with $f l p-17^{\text {prom }}$ :NLS:mCherry. Both reporters are very low or absent in these cells. Bars, $10 \mu \mathrm{m}$.

sequences in the $3^{\prime}$ UTRs of both reporters (Supplemental Fig. S6C) also caused derepression of the akap-1 and cah-3 reporters to a similar extent (Fig. 4C-F). These experiments show that while akap-1 and cah-3 are broadly transcribed, mir-791 specifically represses these genes in the BAG neurons.

\section{akap-1 and cah-3 are functional targets of mir-791}

To test whether these genes are true functional targets of mir-791, we introduced $3^{\prime}$ UTR mutations to disrupt all predicted mir-791-binding sites in the endogenous akap1 or cah-3 loci (as in Supplemental Fig. S6C) and also in the other three top predicted targets. We did this by CRISPR/Cas9-induced homology-directed repair. If any of these genes is a relevant target of mir-791, removal of the binding sites should recapitulate, at least in part, the defect caused by removal of mir-791 itself.

Disrupting the mir-791-binding sites in akap-1 or cah-3 phenocopied the loss of mir-791 in the $\mathrm{CO}_{2}$ response assay (Fig. 5). However, mutations in the 3' UTRs of hbl-1, unc9 , and unc-2 did not affect this behavioral response (Supplemental Fig. S7A-C). We also generated a strain with all five $3^{\prime}$ UTR mutations. These animals display a behavioral defect similar to mir-791(0) (Supplemental Fig. S7D). These data strongly support akap-1 and cah-3 as the two critical targets of mir-791 in the $\mathrm{CO}_{2}$-sensing neurons. In addition, the fact that each target seems to fully account for the effect of mir-791 suggests that they act in the same genetic pathway.

At the molecular level, a higher dose of CAH-3 will affect the kinetics of $\mathrm{CO}_{2}$ and $\mathrm{HCO}_{3}{ }^{-}$interconversion and also likely the local intracellular $\mathrm{pH}$. This might have an impact on signaling through GCY-9 or downstream events. AKAP1 has been shown to generate signaling hubs on the mitochondrial surface (Merrill and Strack 2014). We thus speculate that high AKAP-1 levels in the BAGs might ectopically tether required signaling components away from their site of action.

CAs are expressed in $\mathrm{CO}_{2}$-sensing cells, and their activity has been implicated in $\mathrm{CO}_{2}$ sensing in mammals, frogs, fish, fungi, and plants (Luo et al. 2009; Cummins et al. 2013). Unlike these systems, the BAG neurons of C. elegans seem to express low levels of CAs. Out of six predicted a-CAs in C. elegans, three lack residues important for catalysis. Only CAH-3 and CAH-4 are active in heterologous CA activity assays (Sherman et al. 2012). Based on its published expression pattern, cah-4 seems to be absent from BAG (Bretscher et al. 2011), and here we show that cah-3 is also repressed in these cells. Together, it seems that BAGs do not require high levels of CA activity to fulfill their function. This might reflect the fact that while other systems likely sense $\mathrm{HCO}_{3}{ }^{-}$or $\mathrm{H}^{+}$, BAGs sense $\mathrm{CO}_{2}$ directly (Smith et al. 2013).

\section{A cell type-specific miRNA carves out the expression of broadly expressed genes}

We report that cah-3 and akap-1 are normally repressed within the main $\mathrm{CO}_{2}$-sensing neurons of C. elegans, and their derepression is detrimental to the animal's behavioral response to an important environmental cue. However, because their gene products function in basic

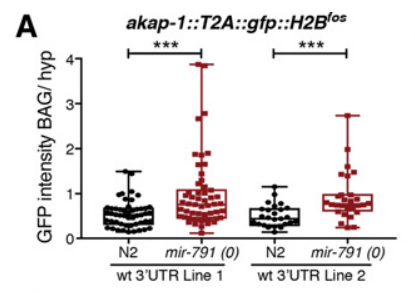

C
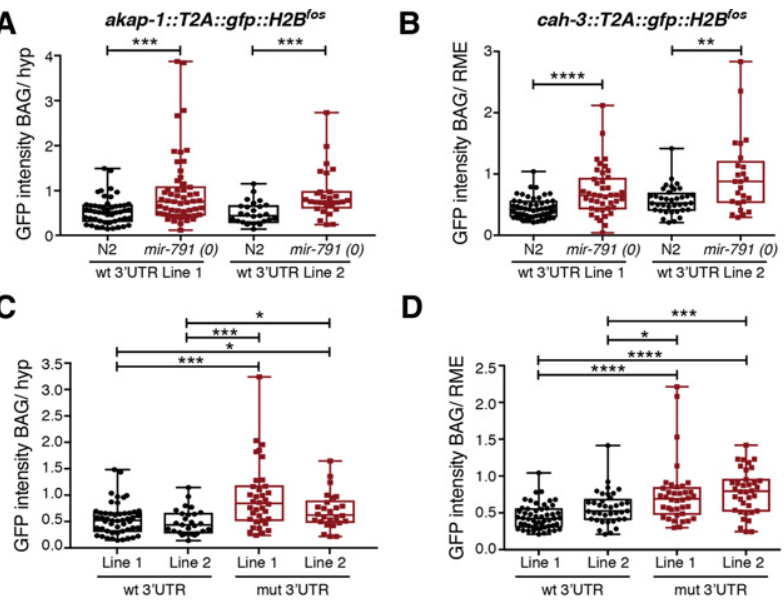

D
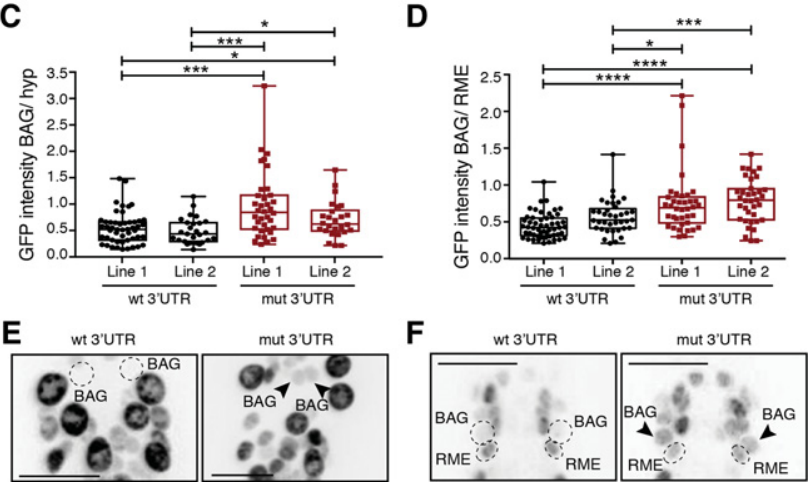

Figure 4. mir-791 represses akap-1 and cah-3 specifically in the BAG neurons. $(A, B)$ Normalized GFP intensity in the BAG neurons of animals carrying akap-1 or cah-3 fosmid reporters with their respective wild-type 3' UTRs in either wild-type (N2) or mir-791(0) animals. Two independent lines were scored per reporter. $(C, D)$ Normalized GFP intensity in the BAG neurons of animals carrying akap-1 or cah-3 fosmid reporters with either wild-type or mutant $3^{\prime}$ UTRs (without mir-791-binding sites) (Supplemental Fig. S6C). Asterisks show $P$-values (Mann-Whitney test) as in Figure 1. $(E, F)$ Representative images of the GFP expression of the reporters quantified in $C$ and $D$. Inverted images are shown. BAG nuclei were localized with flp-17 ${ }^{\text {prom }}$ :NLS:mCherry (not shown); their position is marked by a circle when GFP expression is not visible or arrowheads otherwise. For details on quantification, see the Supplemental Material. Bars, $10 \mu \mathrm{m}$. 
Drexel et al.

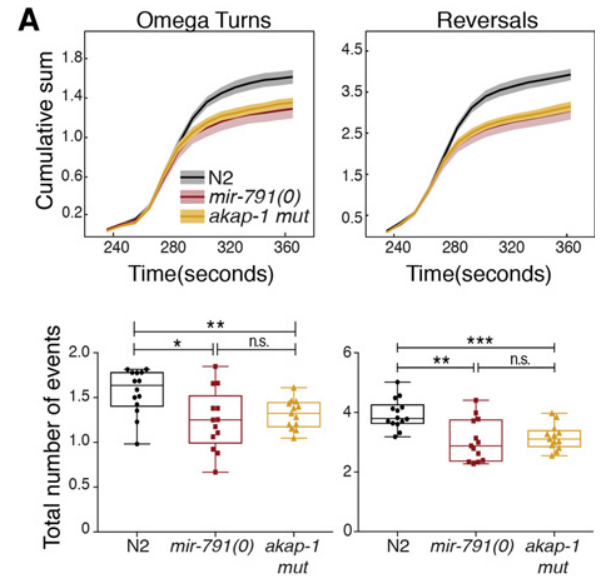

B
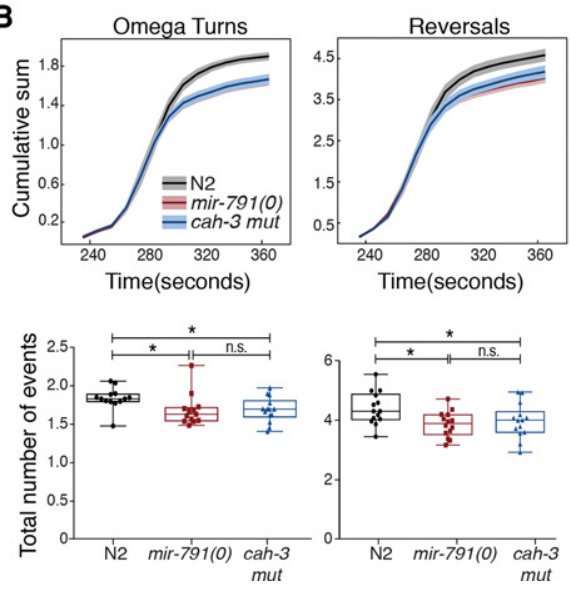

Figure 5. akap-1 and cah-3 regulation accounts for the observed mir791(0) phenotype. (A) Cumulative sum plots and statistical analysis of turning and reversal events (as in Fig. 1) of wild-type (N2) $(n=14)$, mir-791(0) $(n=13)$, and akap-1 3' UTR mutant $(n=13)$ animals. $(B)$ Same as in $A$ but showing comparison with cah-3 $3^{\prime}$ UTR mutant animals. $n=14$ wild-type (N2); $n=14$ mir-791(0); $n=15$ cah-3 3' UTR mutant.

cellular biology, cah-3 and akap-1 are broadly expressed in all major cell types of the animal. For example, down-regulation of akap-1 using RNAi causes embryonic lethality and sterility (Maeda et al. 2001). Here we show that a miRNA expressed exclusively in the $\mathrm{CO}_{2}$-sensing neurons provides the required specificity to the repression of these genes.

Our findings align with observations that so-called ubiquitous genes are often expressed at different levels in different cell types and can be regulated by external conditions. For instance, $\beta$ cells in the pancreas rely on the specific repression of two otherwise ubiquitous genes-a lactate/pyruvate transporter (Mct1) and lactate dehydrogenase (Ldha) - to prevent inappropriate release of insulin when blood glucose is low (Ishihara et al. 1999; Thorrez et al. 2011). While the source of specificity of this repression is unknown, it seems to occur at the transcriptional level. Derepression of Mct1 has been linked to exercise-induced hypoglycemia in humans (Otonkoski et al. 2007); thus, it is important for coordinating systemic responses of a complex organism with changing internal conditions. Here we show that this type of cell-specific repression is necessary in specialized cells that interact with the environment and that precise repression is provided by a specifically expressed miRNA. It had been shown that a miRNA has the capacity to repress a ubiquitous reporter carrying an artificial 3' UTR (Mishima et al. 2009). Here we show that this is a naturally occurring strategy for neuronal specialization with impact on a whole organism.

Two properties of broadly expressed genes and miRNAs suggest that this might be a more general strategy. First, transcriptional control of housekeeping genes is generally more compact and constrained than the modular regulation of developmentally regulated genes (Zeitlinger and Stark 2010; Zabidi et al. 2015). This might make transcriptional regulation more difficult. Second, the fast evolution of miRNAs makes it more likely for novel targeting specificity to arise (Berezikov 2011). Overall, post-transcriptional regulation via miRNAs might be a more likely evolutionary solution to cell-specific repression of broadly expressed genes.

Many miRNAs are expressed with high spatiotemporal specificity, perhaps most noticeably in animals like $C$. elegans, where cell types are represented by as little as single cells. For example, the miRNA lsy- 6 is made and acts in a single neuron (Johnston and Hobert 2003; Cochella and Hobert 2012). In addition to mir-791, we found at least another 26 miRNAs expressed in single or few cell types (K Mahofsky, T Drexel, and L Cochella, unpubl.). We predict that some of these will also regulate broadly expressed genes in specific contexts. Moreover, a highly specialized site of action, rather than redundancy or fine-tuning modulation, may explain to a large extent why we failed to ascribe functions to many miRNAs.

$\mathrm{CO}_{2}$ evokes diverse responses in different animals, ranging from being an attractant when related to food finding to being a repellent when it signals predators or stress (e.g., Cayirlioglu et al. 2008). Therefore, the molecular and neural mechanisms of $\mathrm{CO}_{2}$ sensing and $\mathrm{CO}_{2}$-evoked behaviors are dynamic over evolutionary time even within different isolates of C. elegans (Hallem and Sternberg 2008). We showed that a miRNA can modify this behavior and, given the relatively fast rate of miRNA evolution, propose that mir-791 and others, such as mir-279 in Drosophila (Cayirlioglu et al. 2008), have played a role in the adaptation to different $\mathrm{CO}_{2}$-sensing requirements. Interestingly, while most miRNAs are well conserved among different Caenorhabditis species, mir-791 is relatively divergent. It will be interesting to explore the potential connection between mir-791-mediated regulation and the ability of different species to respond to $\mathrm{CO}_{2}$.

\section{Materials and methods}

Strains

All worm strains were grown under standard conditions (Brenner 1974). A full list of strains used here is in Supplemental Table S2.

\section{Generation of mutant alleles}

Protocol, primers, and sgRNA sequences are in the Supplemental Material and Supplemental Table S3.

\section{Behavioral assays}

Behavioral assays were performed using a similar device previously used for $\mathrm{O}_{2}$ sensory responses (Zimmer et al. 2009) with modifications (see the Supplemental Material). 


\section{Calcium imaging}

Transgenic animals expressing GCaMP6f in the BAG neurons (see the Supplemental Material) were imaged as described previously (Zimmer et al. 2009|. We reported and quantified the fluorescence relative to a baseline: $\Delta F / F=(F-F O) / F O . F O$ is the mean fluorescence of the lower 15 th percentile of all data points in the first $50 \mathrm{sec}$ of recording.

\section{Fosmid recombineering}

Fosmid-based reporters were generated as described previously (Tursun et al. 2009). For details, see the Supplemental Material.

\section{Microscopy}

Quantification of the cah-3 and akap-1 GFP-based reporters in the BAG neurons was performed on $Z$ stacks through the nervous system with a spinning disc microscope. For details, see the Supplemental Material.

\section{Acknowledgments}

We thank A. Stark, O. Hobert, I. Carrera, and Cochella laboratory members for critically reading the manuscript. Some strains were sent by Caenorhabditis Genetics Consortium (National Institutes of Health Infrastructure Program-P40 OD010440). This work was supported by European Research Council (FP7/2007-2013) GA-337161 awarded to L.C., and GA-281869 awarded to M.Z. T.D. is supported by the Austrian Science Fund (FWF, F4323-B09). Basic research at the Institute of Molecular Pathology is supported by Boehringer Ingelheim GmbH.

\section{References}

Aboobaker AA, Tomancak P, Patel N, Rubin GM, Lai EC. 2005. Drosophila microRNAs exhibit diverse spatial expression patterns during embryonic development. Proc Natl Acad Sci 102: 18017-18022.

Arendt D. 2008. The evolution of cell types in animals: emerging principles from molecular studies. Nat Rev Genet 9: 868-882.

Berezikov E. 2011. Evolution of microRNA diversity and regulation in animals. Nat Rev Genet 12: 846-860.

Brenner S. 1974. The genetics of Caenorhabditis elegans. Genetics 77: 71-94.

Bretscher AJ, Busch KE, de Bono M. 2008. A carbon dioxide avoidance behavior is integrated with responses to ambient oxygen and food in Caenorhabditis elegans. Proc Natl Acad Sci 105: 8044-8049.

Bretscher AJ, Kodama-Namba E, Busch KE, Murphy RJ, Soltesz Z, Laurent $P$, de Bono M. 2011. Temperature, oxygen, and salt-sensing neurons in C. elegans are carbon dioxide sensors that control avoidance behavior. Neuron 69: 1099-1113.

Cayirlioglu P, Kadow IG, Zhan X, Okamura K, Suh GSB, Gunning D, Lai EC, Zipursky SL. 2008. Hybrid neurons in a microRNA mutant are putative evolutionary intermediates in insect $\mathrm{CO}_{2}$ sensory systems. Science 319: 1256-1260.

Chalfie M, Sulston JE, White JG, Southgate E, Thomson JN, Brenner S. 1985. The neural circuit for touch sensitivity in Caenorhabditis elegans. J Neurosci 5: 956-964.

Chen T-W, Wardill TJ, Sun Y, Pulver SR, Renninger SL, Baohan A, Schreiter ER, Kerr RA, Orger MB, Jayaraman V, et al. 2013. Ultrasensitive fluorescent proteins for imaging neuronal activity. Nature 499: 295-300.

Cochella L, Hobert O. 2012. Embryonic priming of a miRNA locus predetermines postmitotic neuronal left/right asymmetry in C. elegans. Cell 151: 1229-1242.

Cummins EP, Selfridge AC, Sporn PH, Sznajder JI, Taylor CT. 2013. Carbon dioxide-sensing in organisms and its implications for human disease. Cell Mol Life Sci 71: 831-845.

Hallem EA, Sternberg PW. 2008. Acute carbon dioxide avoidance in Caenorhabditis elegans. Proc Natl Acad Sci 105: 8038-8043.

Hallem EA, Spencer WC, McWhirter RD, Zeller G, Henz SR, Rätsch G, Miller DM, Horvitz HR, Sternberg PW, Ringstad N. 2011. Receptor- type guanylate cyclase is required for carbon dioxide sensation by Caenorhabditis elegans. Proc Natl Acad Sci 108: 254-259.

Ishihara H, Wang H, Drewes LR, Wollheim CB. 1999. Overexpression of monocarboxylate transporter and lactate dehydrogenase alters insulin secretory responses to pyruvate and lactate in $\beta$ cells. J Clin Invest 104: 1621-1629.

Jan CH, Friedman RC, Ruby JG, Bartel DP. 2011. Formation, regulation and evolution of Caenorhabditis elegans 3'UTRs. Nature 469: 97-101.

Johnston RJ Jr, Hobert O. 2003. A microRNA controlling left/right neuronal asymmetry in Caenorhabditis elegans. Nature 426: 845-849.

Langeberg LK, Scott JD. 2015. Signalling scaffolds and localorganization of cellular behaviour. Nat Rev Mol Cell Biol 16: 232-244.

Luo M, Sun L, Hu J. 2009. Neural detection of gases-carbon dioxide, oxygen-in vertebrates and invertebrates. Curr Opin Neurobiol 19: 354-361.

Maeda I, Kohara Y, Yamamoto M, Sugimoto A. 2001. Large-scale analysis of gene function in Caenorhabditis elegans by high-throughput RNAi. Curr Biol 11: 171-176.

Merrill RA, Strack S. 2014. Mitochondria: a kinase anchoring protein 1, a signaling platform for mitochondrial form and function. Int $\mathrm{J}$ Biochem Cell Biol 48: 92-96.

Mishima Y, Abreu-Goodger C, Staton AA, Stahlhut C, Shou C, Cheng C, Gerstein M, Enright AJ, Giraldez AJ. 2009. Zebrafish miR-1 and miR133 shape muscle gene expression and regulate sarcomeric actin organization. Genes Dev 23: 619-632.

Otonkoski T, Jiao H, Kaminen-Ahola N, Tapia-Paez I, Ullah MS, Parton LE, Schuit F, Quintens R, Sipilä I, Mayatepek E, et al. 2007. Physical exercise-induced hypoglycemia caused by failed silencing of monocarboxylate transporter 1 in pancreatic $\beta$ cells. Am I Hum Genet 81: 467-474.

Pierce-Shimomura JT, Morse TM, Lockery SR. 1999. The fundamental role of pirouettes in Caenorhabditis elegans chemotaxis. J Neurosci 19: 9557-9569.

Ringstad N, Horvitz HR. 2008. FMRFamide neuropeptides and acetylcholine synergistically inhibit egg-laying by C. elegans. Nat Neurosci 11: 1168-1176.

Sharabi K, Hurwitz A, Simon AJ, Beitel GJ, Morimoto RI, Rechavi G, Sznajder JI, Gruenbaum Y. 2009. Elevated $\mathrm{CO}_{2}$ levels affect development, motility, and fertility and extend life span in Caenorhabditis elegans. Proc Natl Acad Sci 106: 4024-4029.

Sherman TA, Rongali SC, Matthews TA, Pfeiffer J, Nehrke K. 2012. Identification of a nuclear carbonic anhydrase in Caenorhabditis elegans. Biochim Biophys Acta 1823: 808-817.

Smith ESJ, Martinez-Velazquez L, Ringstad N. 2013. A chemoreceptor that detects molecular carbon dioxide. I Biol Chem 288: 37071-37081.

Stark A, Brennecke J, Bushati N, Russell RB, Cohen SM. 2005. Animal MicroRNAs confer robustness to gene expression and have a significant impact on 3'UTR evolution. Cell 123: 1133-1146.

Supuran CT. 2008. Carbonic anhydrases-an overview. Curr Pharm Des 14: 603-614.

Thorrez L, Laudadio I, Van Deun K, Quintens R, Hendrickx N, Granvik M, Lemaire K, Schraenen A, Van Lommel L, Lehnert S, et al. 2011. Tissuespecific disallowance of housekeeping genes: the other face of cell differentiation. Genome Res 21: 95-105.

Tursun B, Cochella L, Carrera I, Hobert O. 2009. A toolkit and robust pipeline for the generation of fosmid-based reporter genes in C. elegans. PLoS One 4: e4625.

Wienholds E, Kloosterman WP, Miska E, Alvarez-Saavedra E, Berezikov E, de Bruijn E, Horvitz HR, Kauppinen S, Plasterk RHA. 2005. MicroRNA expression in zebrafish embryonic development. Science 309: 310-311.

Zabidi MA, Arnold CD, Schernhuber K, Pagani M, Rath M, Frank O, Stark A. 2015. Enhancer-core-promoter specificity separates developmental and housekeeping gene regulation. Nature 518: 556-559.

Zeitlinger J, Stark A. 2010. Developmental gene regulation in the era of genomics. Dev Biol 339: 230-239.

Zimmer M, Gray JM, Pokala N, Chang AJ, Karow DS, Marletta MA, Hudson ML, Morton DB, Chronis N, Bargmann CI. 2009. Neurons detect increases and decreases in oxygen levels using distinct guanylate cyclases. Neuron 61: 865-879. 


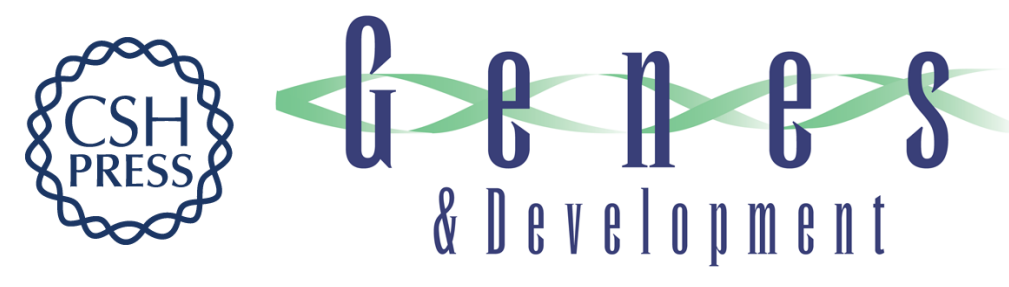

\section{Neuron type-specific miRNA represses two broadly expressed genes to modulate an avoidance behavior in $C$. elegans}

Tanja Drexel, Katharina Mahofsky, Richard Latham, et al.

Genes Dev. 2016, 30: originally published online September 29, 2016

Access the most recent version at doi:10.1101/gad.287904.116

\section{Supplemental http://genesdev.cshlp.org/content/suppl/2016/09/29/gad.287904.116.DC1 \\ Material}

\section{Related Content A sense-able microRNA}

Amy E. Pasquinelli

Genes Dev. September , 2016 30: 2019-2020

References This article cites 35 articles, 13 of which can be accessed free at:

http://genesdev.cshlp.org/content/30/18/2042.full.html\#ref-list-1

Articles cited in:

http://genesdev.cshlp.org/content/30/18/2042.full.html\#related-urls

Creative This article, published in Genes \& Development, is available under a Creative Commons

Commons License (Attribution 4.0 International), as described at

License http://creativecommons.org/licenses/by/4.0/.
Email Alerting Receive free email alerts when new articles cite this article - sign up in the box at the top
Service right corner of the article or click here.

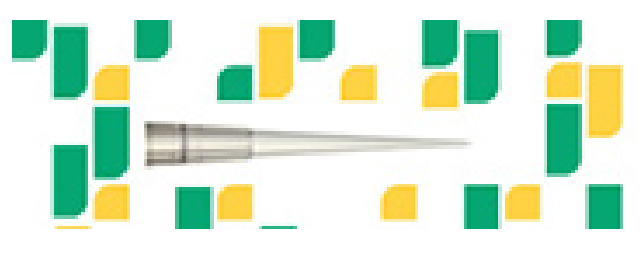

Focused on your science. 